Cotirleț A. ${ }^{1,2}$, Tincu E. ${ }^{1}$, Coşa Raluca ${ }^{1}$, Popa E. ${ }^{1}$, Gavril Laura $^{3}$,Unc O. ${ }^{4}$, Iordache I. ${ }^{4}$

\title{
Laparoscopic repair for perforated duodenal ulcer
}

\author{
${ }^{1}$ Emergency Hospital of Moinesti \\ 2 "Vasile Alecsandri" University of Bacau \\ 3 "Gr.T. Popa" University of Medicine and Pharmacy \\ ${ }^{4}$ Ovidius University of Constanta, Faculty of Medicine
}

\begin{abstract}
Perforated peptic ulcer (PPU), despite antiulcer medication and Helicobacter eradication, is still the most common indication for emergency gastric surgery associated with high morbidity and mortality. Perforated peptic ulcer is a common abdominal disease that is treated by surgery. The development of laparoscopic surgery has changed the way to treat such abdominal surgical emergencies but there is no consensus on whether the benefits of laparoscopic closure of perforated peptic ulcer outweigh the disadvantages such as prolonged surgery time and greater expense. However we can say that laparoscopic repair is a viable and safe surgical option for patients with perforated peptic ulcer disease and should be considered with the necessary expertise available.
\end{abstract}

Keywords: Peptic ulcer, Perforation, Raphy, Laparoscopic treatment

Adrian Cotirlet MD, PhD, Prof. ,

Emergency Hospital of Moinesti , Surgery Department,

Str. Zorilor nr. 1, 605400, Moineşti, Bacău, România

Tel/fax: 0744507926/0234362698

e-mail: spitalmoinesti@bacau.astral.ro

\section{Introduction}

The first series of cases with duodenal perforated ulcer belongs to Travers being published in 1817, Mikulicz describing for the first time in 1884 the surgical technique. However, the first operation with favorable evolution was made in 1892 by Kriege. Though this pathology is a relatively common emergency and a frequent cause of death in elderly patients, there is currently controversy about the treatment of this disease $(1,2)$.

Eradication of Helicobacter Pyilori resulted in significant reduction of the prevalence of cases of peptic ulcers (3). However, the number of patients requiring emergency surgery remained relatively the same, certain selected cases responding positively to the adjuvant non-surgical treatment $(4,5)$. Various techniques were used in surgical treatment including stapled omental patch (6), gastroscopy aided insertion of the ligamentum teres (7) or omental plug (8). Laparoscopic suture of perforated duodenal ulcer was performed for the first time in 1990 by Nathanson et al (9), benefiting from advantages such as shortening the period of hospitalization, minimal postoperative pain, quick social reinsertion, cosmetic 
advantages (10). In the present moment, the most commonly used procedure for laparoscopic repair of perforated duodenal ulcer is the simple suture with or without omental patch, the laparoscopic approach being indicated in any case of suspected duodenal perforation. Contraindications for the laparoscopic technique include high risk patients (ASA class IV), massive ilesu and advanced purulent peritonitis.

\section{Surgical technique}

It would be preferable to use an advanced laparoscopic insufflator with a minimum of $16 \mathrm{l} /$ min, and irrigation-aspiration unit at least 2-4 1/ min. The instruments are similar to those used in most laparoscopic procedures. A $0^{\circ}$ laparoscope is commonly used, but a $30^{\circ}$ laparoscope may be useful to see better a perforated duodenal ulcer placed on the superior surface of the duodenum. The other instruments necessary for this operation are: 2 atraumatic graspers, 2 needle holders, suctionirrigation device, scissors, liver retractor Endotracheal anaesthesia is generally used and intravenous antibiotic therapy should be done before insufflation. The patient will be placed in dorsal decubitus with legs straight and spread out, right arm abducted to 90 degrees, left arm being positioned at the side of the body. The surgeon stands between patient's legs nd the assistant to the patient's left, the monitor being placed at the patient's right shoulder. The pneumoperitoneum can be made by the Veress needle supraumbilical, infraumbilical or in Palmer's point in left. A CO2 intraabdominal pressure between 8 and $12 \mathrm{mmHg}$ is usually sufficient to realize enough room to work properly. The $10 \mathrm{~mm}$ optical trocar is inserted through a $10 \mathrm{~mm}$ incision made in the third distal part of the xipho-umbilical line. One operating trocar of $5 \mathrm{~mm}$ trocar is inserted paraumbilical on the right on the midclavicular line and the other $5 \mathrm{~mm}$ or $10 \mathrm{~mm}$ working trocar is placed paraumbilical on the left on the midclavicular line, the trocar for the liver retractor being placed in the epigastric region.(figure 1).

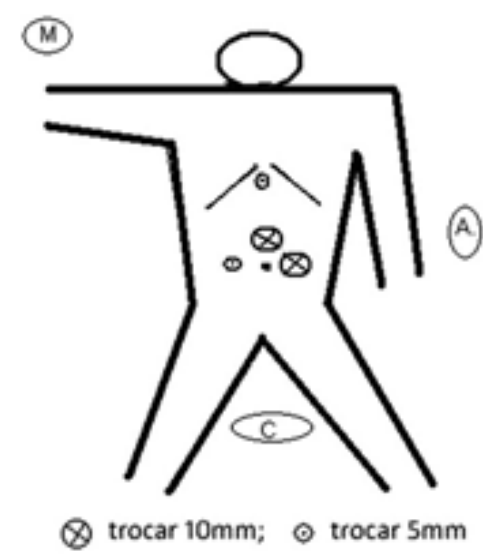

Figure 1: Patient, surgical team and trocars position

A three trocar technique can be used, the liver being retracted with the help of a percutaneous suture that suspends the round ligament of the liver toward the upper left side of the abdomen. The optic is inserted through the supraumbilical trocar and the abdomen is explored to identify the perforation and to assess the magnitude of peritonitis (figure 2).

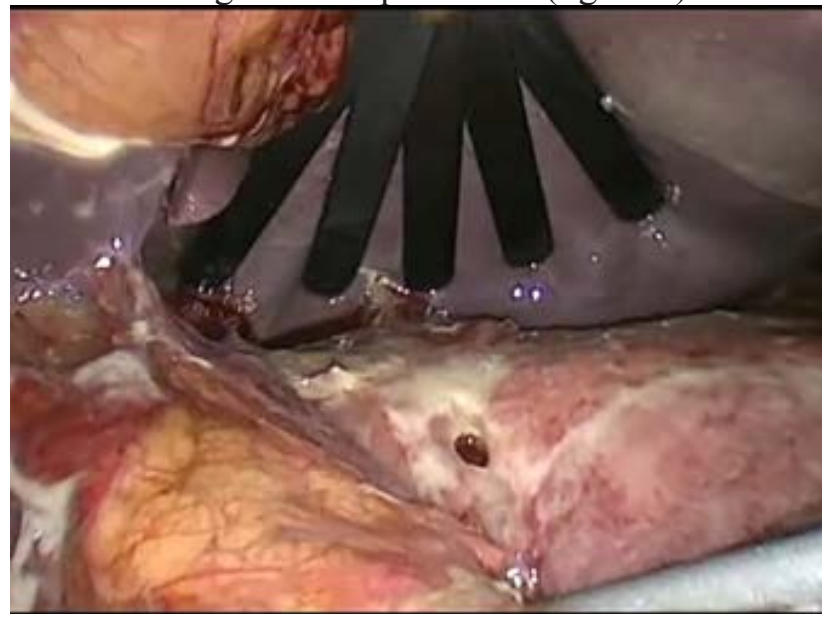

Figure 2: Anterior duodenal perforated ulcer

Bacteriological samples are done and sends immediately to the laboratory. Next step is cleaning the abdomen by irrigation and aspiration with warm saline solution (figure 3). Each quadrant is cleaned methodically, starting at the right upper quadrant, going to the left, moving down to the left lower quadrant, and then finally over to the right. The tilt of the operating table should be adapted as necessary. 
Special attention should be given to the rectovesical oe rectouterine pouch and to the intestinal loops.

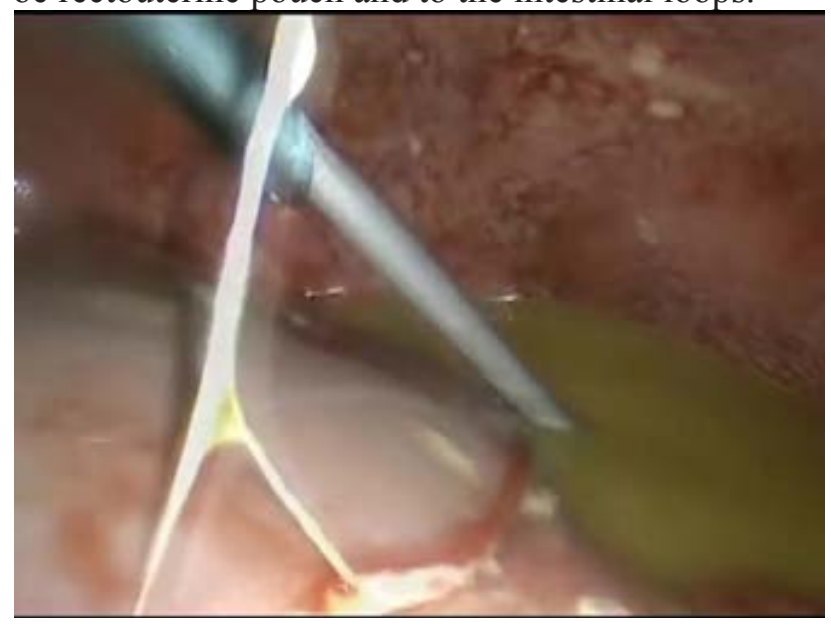

Figure 3: Lvage/aspiration of the peritoneal cavity

Fibrous membranes are removed as much as possible, since they might contain bacteria. Once the abdominal cavity is cleansed the surgeon can proceed to treat the perforation. There are several options for doing that but only 2 of them are utilized more frequently The most common technique is suturing the perforation with standard stitches. Suturing is realized with $2 / 0$ or $3 / 0$ slowly absorbable or nonabsorbable sutures. Interrupted sutures are used and usually two or three stitches are placed in a transversal manner over the perforation focused on the pyloroduodenal axis. Perforations under $5 \mathrm{~mm}$ in diameter can be sealed with a " $Z$ " type suture passed at minium $5 \mathrm{~mm}$ from the lesion borders (figure 4).

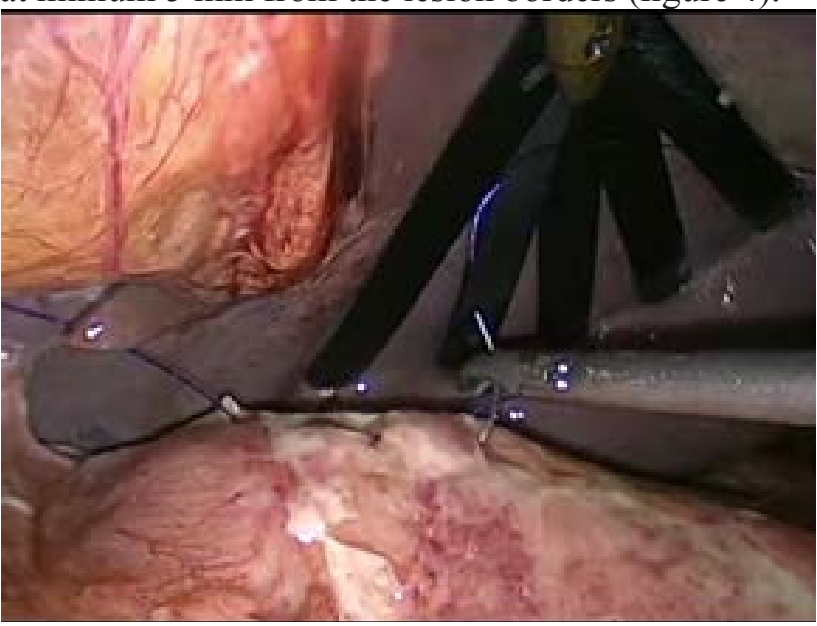

Figure 4: "Z" type suture of the perforation
Once the perforation is sealed, a small fragment of the greater omentum can be fixed over the suture line using the upper thread which was left loose after making the knot (figure 5). Some surgeons prefer to use instead of omental patch fibrin glue which is spread over the suture.

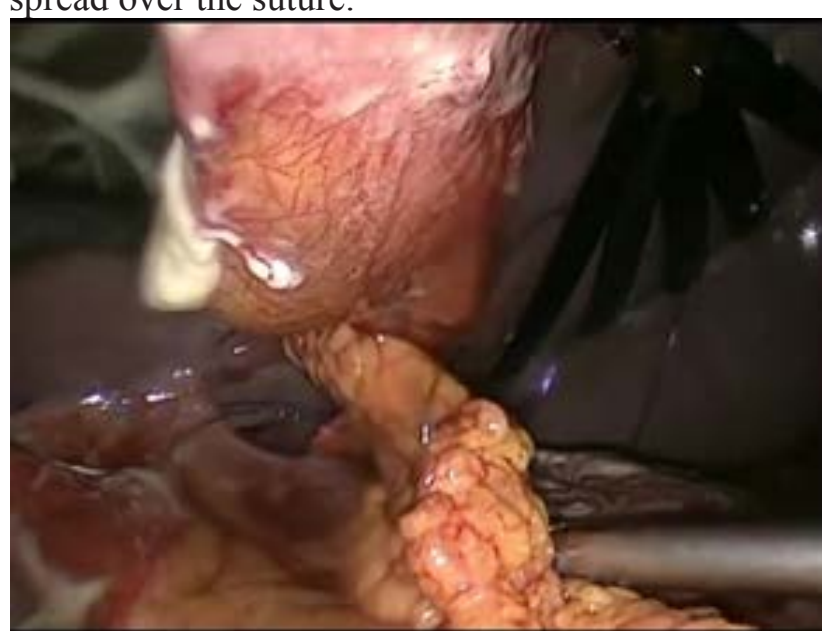

Figure 5: Epiploonoplasty of the suture

When is difficult to approximate the edges of the ulcer, as is the case with chronic callous ulcers, sutures of bigger caliber ( 0 or 1$)$ must be used in order to avoid cutting the duodenal wall. Tthe second most used technique is the closure of the perforation with an omental patch (Graham patch). This procedure is usually utilized for perforations that passes $5 \mathrm{~mm}$ in diameter. A fragment of greater omentum is placed over the perforation and the surgeon sutures it with several interrupted sutures.

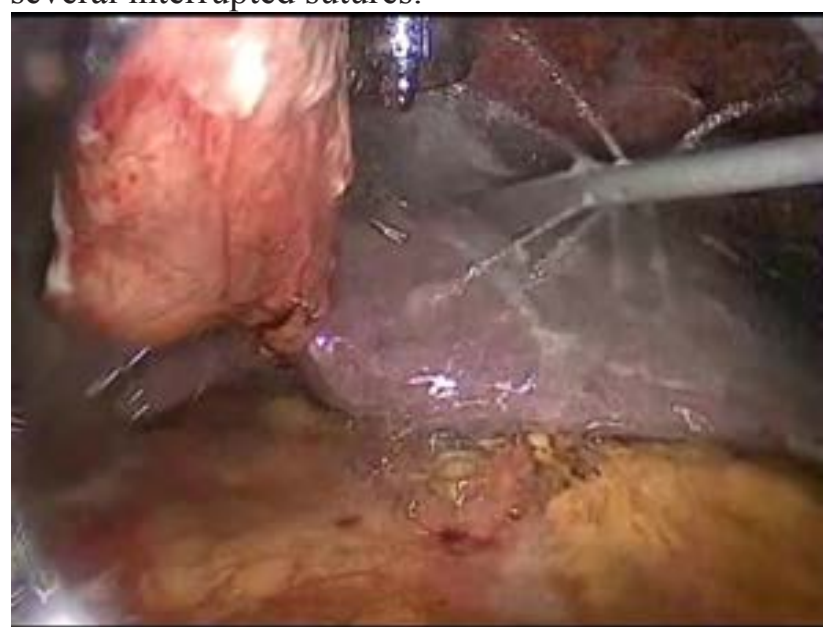

Figure 6: Lavage of abdominal cavity 
Alternative options to seal the perforation may include the use of biological glue and sponge plug as a plasty with the round ligament. The peritoneal lavage is continued after the suture, generally about 4 to 6 liters of warm saline solution being used (figure $6)$.

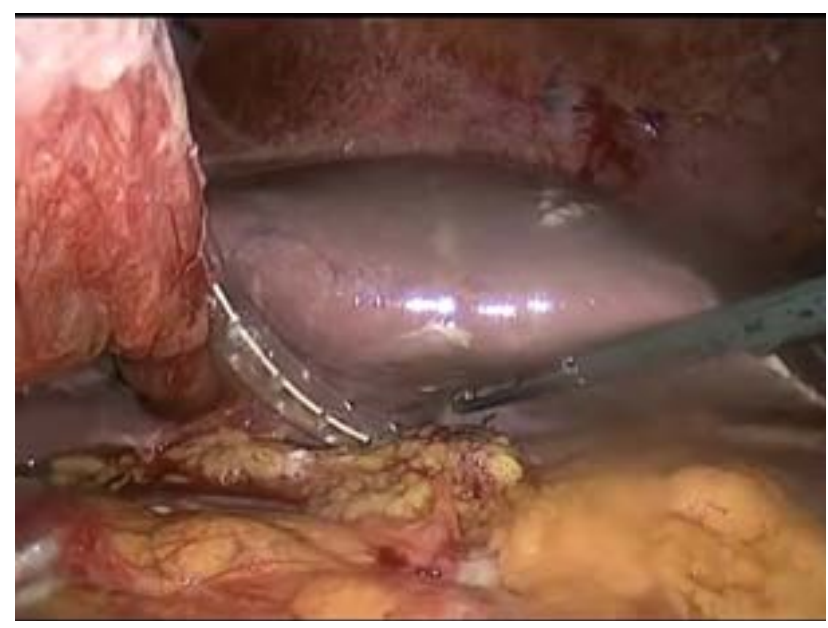

Figure 7 : Insertion of the subhepatic drain

The drainage of the peritoneal cavity is performed using silicone drains. One drain si placed in the subhepatic region (figure 7) and another drain is inserted in the pouch of Douglas (figure 8) Before ending the operation the abdomen must be examined for any possible bowel injury or bleeding. Trocars are removed one after the other and hemostasis of the trocar sites is checked.

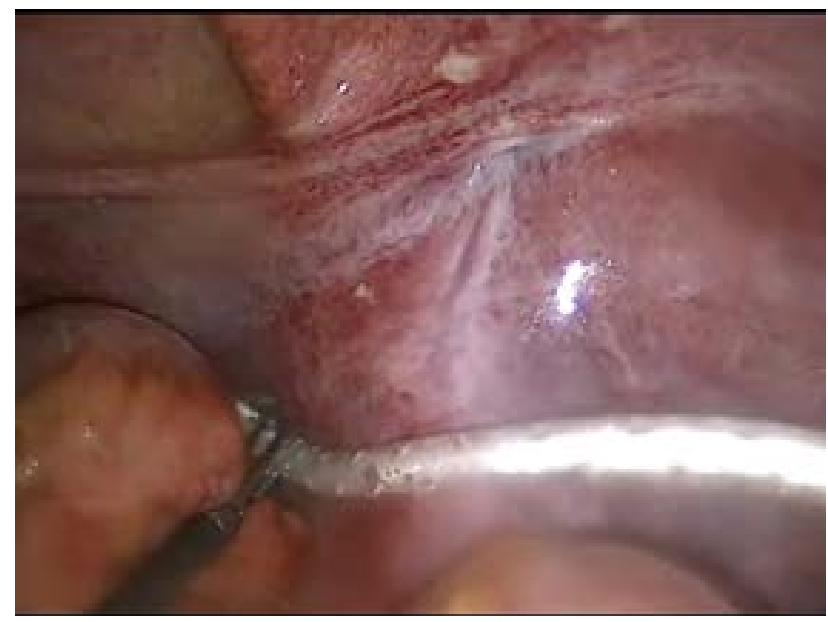

Figure 8: Insertion of the drain in the pouch of Douglas
The telescope is removed, the exufflation of the gas from the abdominal cavity is performed and the trocars are removed one after the other. The skin is closed using staples or intradermic sutures.

\section{Postoperative management}

The patient may have slight pain initially but usually resolves with mild pain killers. Intravenous $\mathrm{H} 2$ receptor antagonists or proton pump inhibitors are given intravenously and then orally once infusions are stopped. Intravenous antibiotic therapy is maintained for minimum 3 days and after that the treatment for Helicobacter Pylori is initiated. The nasogastric tube is removed once peristalsis resumes and the food intake is then restored. Drains are removed once the effluent is less than $100 \mathrm{ml}$ per day (usually after 1 to 3 days). When suturing is difficult or bowel function is resumed late, the gastric tube can be left in place longer.If no complications occures the patient may be discharged 3 to 6 days after operation. Control gastroscopy is performed usually 4 to 6 weeks after the operation.

\section{Postoperative complications}

The postoperative complications can be general or/and specific. The general complications belongs to cardiovascular and pulmonar pathology. The specific complications can occur early after the operation (suture leak, bleeding from an unknown ulcer) or later (stenosis, ulcer recurrence). 
randomized controlled trials are necessary for establishing the advantages of the procedure for patients with multiple risk factors.

Inadequate ulcer localization and large ulcer size are the most common reasons for conversion Other possible reasons are: the posterior pyloroduodenal ulcer, infiltration and fragility of ulcer edges, associated bleeding and hemodinamic instability. experience

The Emergency Hospital of Moinesti

Between 2009 and 2013, in our surgical department a laparoscopic approach was attempted in 30 patients with perforated duodenal ulcer. There were 27 men and 3 women with ages between 15 and 85 years. We used simple suture of the perforation for 21 cases and suture with epiploonoplasty for 9 patients. There were 4 conversions to open technique for the following reasons: fragility of ulcer edges (2 cases), diameter of the perforation over $1 \mathrm{~cm}$ (1 case) and inadequate localization of the ulcer ( 1 case). The operating time varied between 25 and 74 minutes, the postoperative complications consisted in: suture leak ( 1 case), subhepatic abscess ( 2 cases), pulmonary complications ( 3 cases), wound infections ( 2 cases). The hospital stay was between 3 to 25 days, most patients being discharged 5 days after surgery.

\section{Conclusions}

Advances in the medical treatment of peptic ulcer disease and Helicobacter pylori eradication have led to a significant decline in peptic ulcer prevalence and a dramatic decrease in the number of elective ulcer surgeries performed. Minimally invasive surgery has gained a highly expanding role in gastrointestinal surgery since the introduction of laparoscopic cholecystectomy. In the last few years, the role of laparoscopic surgery in management of perforated duodenal ulcer has gained more popularity among laparoscopic gastrointestinal procedures, the technique being considered safe and feasible for patients with maximum 1 risk factor. However,

\section{References}

1. Koo J., Ngan Y.K. \& Lam S.K. (1983). Trends in hospital admissions, perforation and mortality of peptic ulcer in Hong Kong from 1970 to 1980. Gastroenterology. 84(6), 1558-62.

2. Alagaratnam T.T. \& Wong J: (1988). No decrease in duodenal ulcer surgery after cimetidine in Hong Kong. J Clin Gastroenterol, 10:25-27

3. Hopkins R.J., Girardi L.S. \& Turney E.A. (1996). Relationship between Helicobacter pylori eradication and reduced duodenal and gastric ulcer recurrence: a review. Gastroenterology. 110, 1244-1252.

4. Lam S.K., Byth K. \& Ng M.M. (1992). Perforated peptic ulcer in Hong Kong and New South Wales. $J$ Gastroenterol Hepato. 17,508-511.

5. Crofts T.J., Park K.G.M. \& Steel R.J.C. (1989) A randomized trial of non-operative treatment for perforated peptic ulcer. N Engl J Med 320,970-3.

6. Darzi A., Cheshire N.J. \& Somers S.S. (1993). Laparoscopic omental patch repair of perforated duodenal ulcer with an automated stapler. $\mathrm{Br} J$ Surg. 80, 1552.

7. Costalat G., Dravet F. \& Noel P. (2001). Coelioscopic treatment of perforated gastroduodenal ulcer using the ligamentum teres hepatis. Surg Endosc 5.154-155

8. Pescatore P., Halkic N., Calmes J.M. (1998) Combined laparoscopic-endoscopic method using an omental plug for therapy of gastroduodenal ulcer perforation. Gastrointest Endosc 48,411-414

9. Nathanson L.K., Easter D.W. \& Cuschieri A. Laparoscopic repair/peritoneal toilet of perforated duodenal ulcer. Surg Endosc. 4,232233.

10. Varcuş F., Lazăr F. Beuran M. et all. (2013) Laparoscopic treatment of perforated duodenal ulcer - a multicenter study. Chirurgia. 108(2), $172-6$ 